\title{
REPORT ON INTERNATIONAL MEDICAL SOCIETY OF PARAPLEGIA 31ST ANNUAL SCIENTIFIC MEETING 8 TO 10 SEPTEMBER 1992
}

\section{S Pullen}

\section{INTRODUCTION}

The congress took place in Sitges, just $30 \mathrm{~km}$ from Barcelona in Spain. The venue was the Gran Sitges Hotel which provided excellent congress facilities. Delegates from America, Australia, Great Britain, Europe, Middle and Far East and South America attended this congress. Delegates and speakers included orthopaedic surgeons, paraplegists, urologists, neurologists, traumatologists, surgeons, psychologists, social workers, radiologists, physiotherapists and occupational therapists. This emphasises the multi-faceted specialist attention and coordinated teamwork required for total and competent treatment of spinal cord injuries. For the physiotherapist working in this field this reinforces the fact that knowledge and understanding of each specialist's involvement in the treatment of the spinal cord injured is vital if we are to function as a competent member of the team. This report attempts to highlight information which is important from a physiotherapist's point of view so that we can clinically apply what we have learned.

\section{Spinal Cord Lesions in Childhood}

Most researchers defined childhood spinal cord lesions as being those occurring before the age of 16 . The most common cause of injury is motor vehicle accidents, followed by falls, but in America sports injuries and violence are more common.

Spinal deformities are a common complication. Apple stated that scoliosis is eight times more common in paediatric than in adult spinal cord injuries. Alcaraz, et al reported a $74 \%$ incidence of scoliosis in children older than 6 years. At Stoke Mandeville Hospital a retrospective study of 299 children older than 16 years of age found that the degree of scoliosis was greater in paraplegics (approx $33^{\circ}$ ) than in tetraplegics (approx $16^{\circ}$ ) and that $77 \%$ of the scoliotic curves were greater than $10^{\circ}$.

Lung function and thoraco-lumbar bracing was studied by Williams $e t$ al who found that bracing significantly decreased lung function and they emphasised that these effects need to be considered, when providing thoraco-lumbar bracing to prevent deformity in children with already significantly compromised lung function.

As spinal deformity, especially scoliosis, is commonly presented to the physiotherapist for exercises and/or bracing, the findings of these studies are important considerations in the application of physiotherapeutic treatment and advice.

\section{Spinal Cord Lesions in Developing Countries}

Representatives of spinal cord injury units from Spain, Armenia, Brazil, Algeria, Thailand, and South Africa presented the history, development and functioning of their units. Shrosbree presented "The Management, Rehabilitation, Re-integration, and Re-employment of Spinal Cord Injured South African Mineworkers - First World Medicine in a Third World Country" (Ithuseng Spinal Rehabilitation Centre, Welkom). Patients here are faced with the difficulties of a third world rural environment at their homes and the management of this population requires specific considerations. These facts influence the approaches of the various team members. Physiotherapists, in particular, strive towards teaching and achieving functional walking where possible, learning to fall in calipers and to stand up again and to climb stairs in calipers, so that where the terrain is too rough for a wheelchair, the patients can still manage to be mobile in some way, especially in an emergency situation.

\section{Neurological Changes after Conservative versus Surgical Treatment}

There was much debate regarding conservative versus surgical treatment. There appears to be no difference in neurological outcome between the two approaches. The type of surgical fixator being used internationally eg. Harrington rods, is inhibitory as regards flexibility of the spine. It was also suggested that surgery should be performed by skilled, experienced surgeons only. One definite advantage of surgical treatment is a shorter hospital stay and rehabilitation period.

An important orthopaedic point made by Cassar-Pullicino et al was that a unilateral facet dislocation of the cervical spine once reduced is unstable and requires surgery after reduction.

\section{Recent Advances in the Investigation of Spinal Cord Injuries.}

Many scientific clinical trials were presented in this session. Some of the highlights include the following:

Transected cat spinal cord can be surgically reconstructed using a fluid collagen matrix to bridge the proximal-distal cord stumps. An intact pedicle omentum is then placed on the bridge to increase its blood supply. A video was shown to demonstrate the cat attempting to walk with some recovery in its hind legs.

In Japan, an experimental study on microsurgical reconstruction of the cauda equina involved ventral root crossing and dorsal root grafting. The caudal nerves showed satisfactory regenerative progress similar to that of peripheral nerves by using electro-physiological and histopathological investigations. The authors stated that these techniques may be useful in the treatment of crush injuries and caudal nerves and for reconstruction of bladder function in conus medullaris injuries.

Boundary dysaesthetic pain in paraplegic patients is a recurring subject of interest. Wiese, used "in-situ" hybridisation and immunocytochemistry to show that there is a dramatic increase in neuronal expression of Growth Associated Protein (GAP-43) after spinal cord lesion. This is detected in the dorsal root ganglions, and in preganglionic sympathetic neurons of the intermediolateral nucleus within the spinal cord segments, located immediately rostral to the lesion side. These data may give morphological evidence as a structural basis for the boundary dysaesthetic pain after spinal cord injury.

For physiotherapists, the Functional Electrical Stimulation(FES) System for ambulation in paraplegics has been a major step forward in gait training. Byron Marsolias has investigated the use of FES in tetraplegia. A Frankel C6-7 tetraplegic patient was implanted with electrodes in the quadriceps, hamstrings, gluteus maximus, and paraspinal muscles. The patient achieved independent transfer using eight electrodes with energy expenditure well tolerated. It appears that this technique may prove valuable to many tetraplegics in reducing pressure problems and stress on attendants and in eliminating the need for transfer assisted devices. FES for paraplegics is being used more frequently. The average maximum distance covered is 1300 metres at an average speed of one metre per second. Electrodes are now also being implanted subcutaneously.

\section{Proffered Papers}

Surgical rehabilitation of the upper limb in tetraplegia was presented by surgeons from Sweden, Spain, and France.

Arvid Ejeskar emphasised the constant evolution of methods used and the fact that spasticity is no longer a contraindication. He described how the posterior deltoid and biceps muscles are used to create a triceps muscle and how brachioradialis is used to obtain wrist extension.

Rabischong, et al compared the length-tension relationship of the transferred posterior deltoid in tetraplegics to that of the triceps in normal subjects. The peak torque was recorded at $130^{\circ}$ in patients, compared to $70^{\circ}$ in the control group. There were tremendous differences within the patient group which has been attributed to the initial tension given by the surgeon in the absence of a device to control this variable. The author's protocol is a full stretch of the transferred muscle during surgery and then to immobilise the shoulder in $90^{\circ}$ of abduction and the elbow at $0^{\circ}$. Shoulder abduction is then increased by $30^{\circ}$ per week. From a physiotherapists point of view it is wise to follow this regime in order to achieve the best functional results. 
An interesting paper was a comparison of two methods of weaning persons, with high tetraplegia, from ventilators. There were 74 attempts to wean the 52 subjects using either Synchronised Intermittent Mandatory Ventilation (SIMV) or Progressive Ventilator Free Breathing (PVFB). There was a statistically significant difference ( $p$ $<0.001$ ) between the success rate of SIMV (32\%) and PVFB (77\%). Twelve cases were successfully weaned on PVFB after having failed SIMV attempts. PVFB was more successful if used early.

Very applicable to physiotherapists was a study by Shea et al who concluded that maximal exercise prior to standing or sitting in a wheelchair increases the sensitivity of the carotid cardiac baroreflex for 24 hours after exercise. This is useful as a treatment in the acute management of orthostatic hypotension.

The American Spinal Injury Association (ASIA) presented "Standards for Neurological and Functional Classification of Spinal Cord Injury" which includes definitions, neurological examination, sensory and motor scores/levels, ASIA Impairment Scale, clinical syndromes and the Functional Independence Measure (FIM). This is vital information in order for us to perform neurological assessments which are internationally accepted.

\section{Posters}

Two poster presentations were found to be applicable to physiotherapists. The first was entitled "Psychological Therapy of Spinal Spasticity" by Strubreither et al, who showed how relaxation techniques, performed in groups, decreased spasticity. The second was "The Relationship of Para-articular Ossification to Passive Movements in Paraplegic Patients" by Daud $e t$ al. They concluded that there is a relationship between passive movements which are commenced more than seven days after injury and para-articular ossification. Their treatment protocol at Stoke Mandeville Hospital for acute para-articular ossification is one full range of movement twice per day for six weeks, then once per day.

\section{Impressions of the IMSoP Congress}

Firstly, it was obvious that there is a desperate need for clinical research in the field of physiotherapy in spinal cord injuries.

Secondly, it was evident that internationally, very large numbers of subjects are used in studies. Even 50 or 100 is little. Many studies include hundreds and some even thousands of patients.

The American and British Papers were especially well presented and consisted of interesting and vital subject matter. In fact if one follows their example of careful research design, precise methodology, clearly defined variables, and simple and concise presentation then international standards are easily achieved.

In the light of these observations it becomes very clear that the Ithuseng Spinal Rehabilitation Centre (ISRC) is the ideal place to conduct research. One can easily attempt a retrospective study or a clinical trial, as all the necessary resources are available. As a spinal unit there is the potential to offer much in the way of research, both to national and international medical personnel, in the field of spinal cord injuries.

Another interesting observation is the time required for rehabilitation in other spinal units internationally. Some units quote two or three months for rehabilitation! However it is obvious that their rehabilitation may not include functional walking as far as possible, clinical psychotherapy and sexual counselling, job preparation, work assessment and job placement. At ISRC these aspects are emphasised as well as giving particular attention to the patient's home environment which is in a rural setting. Thus, at ISRC, the rehabilitation period is longer than that of some other units.

\section{Conclusion}

In order for any spinal unit to function optimally, the latest information needs to be shared and gathered by all specialists and therapists working in the field of spinal cord injuries. The IMSoP congress was just that, and provided all delegates, be they doctors, specialists, therapists, psychologists or social workers, with some piece of vital information which they will be able to apply in clinical practice for the good of the spinal cord injured patient.

\section{SPECIALISATION AND THE COLLEGE OF PHYSIOTHERAPISTS OF SOUTH AFRICA}

Specialisation is necessary to identify and to provide formal recognition to physiotherapists with advanced clinical knowledge, experience and skills as well those who have knowledge of research in a specific area of clinical practice.

The benefits of specialisation to the patient are numerous and include access to care from an acknowledged expert. The patient should receive more efficient care and may benefit from the expert's knowledge of up to date research in the field. Preventative care will be emphasised and the specialist will be able to disseminate the latest developments in their specialist field to other members of the profession to the benefit of patients and colleagues.

Incentives to become specialists include recognition of achievement, the opportunity to work in a specialised area with colleagues sharing the same interests and increased job satisfaction.

With the establishment of specialisation we will have to address the issue of the specialist versus the generalist.

The South African health care system is in a state of change with much more emphasis on delivering adequate care to communities where physiotherapy services have in the past not been adequate. For the majority of South Africans adequate physiotherapy services are still not provided. How then do we justify the establishment of specialised services? The profession could look to the specialists to identify the needs of the communities in their area of expertise and to advance suggestions on optimising physiotherapy services.

The specialist could actively participate in research. This is essential to validate the practice of physiotherapy, to establish a strong identity and to communicate this identity to others.

\section{THE SPECIALISATION PROCEDURE}

The specialisation process has been finalised in the following areas:

- Orthopaedic Manipulative Therapy

- Cardiopulmonary Rehabilitation

- Paediatric Neurology and Developmental Disabilities

\section{Basic Qualifications for Admission to Specialisation}

A Four year B Sc Physiotherapy or B Physiotherapy degree is required unless the candidate holds a post-graduate qualification in physiotherapy.

\section{Application for Admission}

When applying for admission with a basic qualification only, the candidate has to submit the following:

- certified copy of the degree

- academic record supplied by the relevant university

- documentary evidence of at least two years' clinical experience in the relevant field

- certificates of any successful completion of continued physiotherapy education courses in the relevant field

With a post-graduate degree and certified qualifications in Neurodevelopmental Therapy, OMT 1 or an Emergency Medical Attendants' course, the candidate can apply for admission to Stage II. The candidate must submit the following:

- the same information as for Stage I

- certificates in Neurodevelopmental Therapy or Orthopaedic Manipulative Therapy or Emergency Medical Attendants Course (depending on the desired area of specialisation)

- certified copy of an Honours degree

- academic record for the post-graduate degree, supplied by the relevant university

- any other information such as publications, papers delivered and teaching experience that may support the application.

With a past-graduate degree a candidate who wishes to apply for Stage III must submit the following:

- the same information as required for Stage 1

- documentary evidence of at least four years' clinical experience in the relevant field

- certified copy of a Master's degree in the relevant field

- a copy of the Master's thesis or, if by course-work, details of the curriculum followed

- details of courses followed in the area of desired specialisation

- details of research experience, publications, papers delivered or teaching in the relevant field. (This information is not essential but may be submitted in support of your application)

Interested candidates can obtain application forms, guidelines for specialisation, the structure of the examinations and fees for specialisation from the Registrar of the CPSA. Candidates should state their area of interest for specialisation when applying. Appllcations must be submitted by 30 September 1993 for commencement in January 1994 or by 1 April 1994 for commencement in July 1994.

Completed forms must be retumed to: The Registrar, The College of Physiotherapists of South Africa, Physiotherapy Department, Medical School, 7 York Road, Parktown 2193. 